\title{
Texture Analysis and Bioadhesive of a Hydrogel as Platforms for Herbal Gel in Sports Massage
}

\author{
Sukanjana Kamlungmak ${ }^{1, *}$ Teerapol Srichana ${ }^{2}$
}

\author{
${ }^{1}$ Department of the Thai Traditional Medicine, Faculty of Health and Sport Science, Thaksin University \\ Phatthalung, Thailand \\ ${ }^{2}$ Department of the Pharmaceutical Technology, Faculty of Pharmaceutical Sciences, Prince of Songkla \\ University, Songkhla, Thailand \\ *Corresponding author.Email: ksukanjana@tsu.ac.th
}

\begin{abstract}
This study investigated the texture analysis and bioadhesive of hydrogel prepared by Poloxamer 407 (P407) and crosslinked Poly (vinyl alcohol); cPVA as hydrogels. All hydrogels were characterized by texture analysis. The bio-adhesive force between the pig's skins and hydrogel was assessed using TA.XT plus texture analyzer. It is expected that the blend polymers can provide suitable properties of hydrogel. The formulations were prepared by varying the ratio between P407 incorporated with cPVA. The results indicated that all hydrogel bases were clear soft gels. These properties were dependent on the ratio of the two polymers. The high ratio of cPVA caused more mechanical and bioadhesive hydrogel in appearance. The firmness and cohesiveness of the hydrogel decreased when the p407 decreased whereas the cPVA proportion in the formulation increased, where cPVA enhanced bioadhesive of the hydrogel system. The research also showed the mixture of P407 and cPVA that was suitable for the development in sport massage herbal gel
\end{abstract}

Keywords: Hydrogel, Sports massage, Herbal gel

\section{INTRODUCTION}

Hydrogels are the common pharmaceutical preparation that could be used in various applications including wound healing, cell therapeutics, bone regeneration and the sustained release of drugs[1-2]. Hydrogels are the crosslinked hydrophilic polymer, which could be classified into 2 groups based on crosslinking interaction by either covalent interaction (chemical hydrogel) or noncovalent interaction (physical hydrogel. Cellulose, alginate, chitosan, poly (vinyl alcohol) and poly (ethylene glycol) are example of hydrogel polymer for wound healing[3, 4]. In addition, hydrogel also could be characterized by theirs properties. The first is swelling kinetic or absorbability. The hydrogel could swell up to $99 \%$ $(\mathrm{w} / \mathrm{w})$ water of their dry weights and this property could also be considered as controlling factor for drug release behavior. The second is pore size. Recently, the study reported the hydrogels for the biomedical sciences normally are in range of $30-150$ nanometer. The third is tensile strength. This parameter is represented the mechanical properties of hydrogel through the functional group and processing of hydrogel. The last is biodegradable property, which would provide the alternative mechanism for drug release and also safety. Recently, the previous report showed that the hydrogel could improve the wound healing process by preventing tissue dehydration and accelerated angiogenesis[5].

The product of sport massage in Thai traditional massage are creams, lotions, and ointments that are contenting herbal extract in oil. The disadvantages of creams, lotions, and ointments base are greasiness and stickiness, that are not desirable for sports player. The development of hydrogel is suitable for herbal extract that can improve formulation base and can be use during for post-performance, rehabilitation, or myofascial pain syndrome.

The present study investigated the texture analysis and bioadhesive of hydrogel of the developed formulations as platforms for herbal gel in sport massage. 


\section{METHODS}

\subsection{Materials}

P407 (poly(propylene oxide)-poly(ethylene oxide) - poly(propylene oxide) triblock copolymers) was gifted from Vita company (Bangkok, Thailand). Poly (vinyl alcohol) (PVA) 87-89\% hydrolyzed from Sigma-Aldrich (Dorset, UK) was used in this study with MW 31,000-50,000. Sodium borate was purchased from PC drug (Bangkok, Thailand). Ultrapure water was used for all samples formulation.

\subsection{Preparation of P407 Hydrogels}

The P407 hydrogel was prepared by dispersing the P407 in ultrapure water by mechanical stirring (400 $\mathrm{rpm}$ ). Then the stock solutions were stored in a refrigerator at $4{ }^{\circ} \mathrm{C}$ for at least $24 \mathrm{~h}$. The cPVA hydrogel was done by adding sodium borate into PVA solution. Blending 15 formulations of P407 hydrogel and cPVA hydrogel was done by mixing of P407, and cPVA in ratio of 100:0, 80:20, 60:40, 40:60, 20:80, and $0: 100$.

\subsection{Texture Profile Analysis (TPA)}

All P407-cPVA hydrogels were characterized by texture analysis. In brief, a Texture Analyzer TA. XT Plus (Stable Micro Systems Ltd., Surrey, UK). All P407-cPVA hydrogels were characterized by texture analysis. In brief, a Texture Analyzer TA-XT Plus (Stable Micro Systems Ltd., Surrey, UK) was used for backward extrusion measurements. A disc (20 millimeter diameter) was pushed at a speed of $4 \mathrm{~mm} / \mathrm{s}$ for a distant of $10 \mathrm{~mm}$ into the hydrogel $(30 \mathrm{~g})$ and redrawn. The texture properties (i.e., firmness, cohesiveness, index of viscosity, and consistency) of the hydrogels were determined. The maximal force achieved during the downward movement of the disc represented the firmness. Consistency of the work was required to compress the disc into the hydrogel, whereas cohesiveness was the sufficient force in the upward movement of the disc that represented a measure of the ability of the formulation to adhere on the disc.

\subsection{Bio-adhesion Studies}

The bio-adhesive force between the pig's skins and P407-cPVA hydrogel was assessed using TA. XT plus texture analyzer (Stable Micro Systems, Surrey, England) in the Hold until Time (HUT) mode. The bio-adhesive force between the pig skins and the P407-cPVA hydrogels was assessed using the TA-XT plus texture analyzer (Stable Micro Systems, Surrey, England) in the Hold Until Time mode. The skins were obtained from newborn pigs that had died of natural causes shortly after birth. They were purchased from a local pig farm in Songkhla Province, Thailand. The skins were soaked overnight in phosphate buffered saline solution before the adhesive experiments. The skins were thawed in physiological saline solution containing $0.9 \%(\mathrm{w} / \mathrm{v}) \mathrm{NaCl}$ at $25 \pm 0.5^{\circ} \mathrm{C}$ for $30 \mathrm{~min}$ before the experiment. The skins were then attached to the lower end of a cylindrical probe (diameter $10 \mathrm{~mm}$ ). The P407-cPVA hydrogels were placed below the probe and immersed in a $35 \pm 0.5{ }^{\circ} \mathrm{C}$ water bath. The test was performed by lowering the probe at a constant speed $(0.1 \mathrm{~mm} / \mathrm{s})$ until the skin and sample made contact detected by a triggering force of $25 \mathrm{~g}$. The skin and the sample were kept in contact for $60 \mathrm{~s}$ and no force was applied during this interval. After $60 \mathrm{~s}$, the skin was drawn upwards $(0.1 \mathrm{~mm} / \mathrm{s})$ until the contact between the surfaces broke. During the experiment a force-time curve was recorded from which the work of adhesion (the area under the force-distance curve during the withdrawing phase) and the force of adhesion were calculated.15 The measurements were performed in triplicates and each sample was measured five times.

\section{RESULTS}

\subsection{Texture Analysis of P407-cPVA Hydrogel}

The firmness and cohesiveness of P407-cPVA hydrogels were showed in Figure 14. The firmness and cohesiveness of the hydrogel decreased when the p407 decreased whereas the cPVA proportion in the formulation increased. The $\mathrm{P} 407$ provided the highest firmness compared with the other cPVA-incorporated p407 formulations. The consistency and index of viscosity of the P407-cPVA decreased drastically when the P407 portion decreased (Figure 1). However, the appearance of a smoother gel was observed in the formulations containing cPVA.

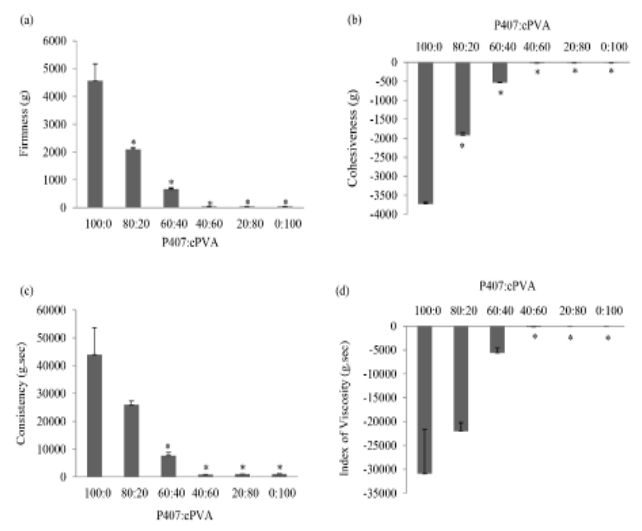

Figure 1 The hydrogel's texture properties express as force and its work: (a) firmness, (b) cohesiveness, (c) consistency and (d) index of viscosity. mean $\pm \mathrm{SD}, \mathrm{n}=3$ *Significant p-value $<0.05$; Compare mean with P407 $23.08 \%$ : cPVA $7.14 \%(100: 0)$ 


\subsection{Bioadhesive Properties of A Modified Thermoresponsive Hydrogel}

The bio-adhesion properties of the mixed hydrogel (P407: cPVA) including the force of adhesion and work of adhesiveness which acted on the bio-mucous surface are presented in Figure 2. The porcine skin was covered over the measurement probe to represent a mucous membrane. Force was applied for $1 \mathrm{~min}$ before the probe was pulled back to determine the bioadhesion parameters. The adhesiveness was first affected by the proportion of cPVA. The addition of cPVA in the hydrogel elevated the bio-adhesive force and work by $20 \mathrm{~g}$ of the $80: 20$ and $60: 40$ hydrogels compared to P407 alone (Figure 2). Then the adhesive force suddenly dropped $(\sim 5 \mathrm{~g})$ as the total water content in the formulations increased according to the high-water content in the cPVA part (only $7 \%$ of polymer concentration). Therefore, the hydrogel properties disappeared by the addition of water.

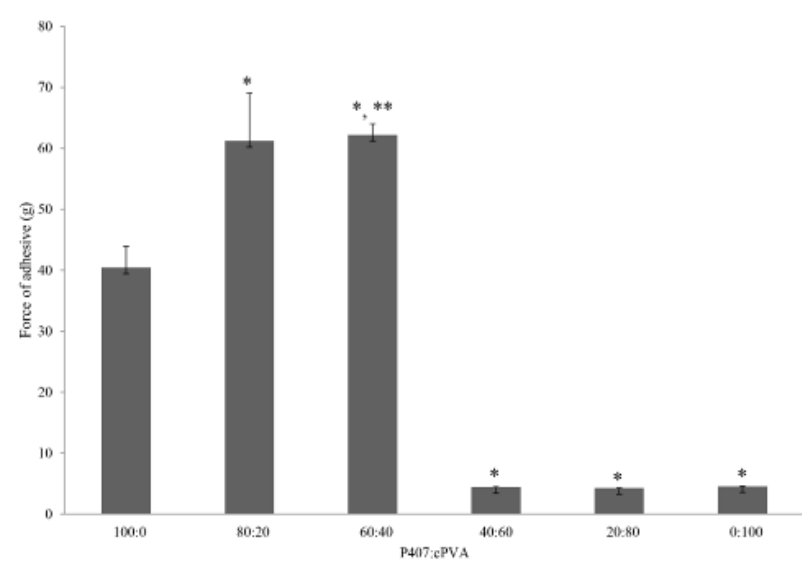

Figure 2 Bio-adhesive force comparison of varying P407: cPVA proportion act on porcine skin at $35^{\circ} \mathrm{C}$. Mean \pm SD, $n=3 *$ Significant $\mathrm{p}$-value $<0.05$

\section{DISCUSSION}

The results indicated that all hydrogel bases were clear semi-solid hydrogels because the hydrogel may be several types such as solid semi-solid and lipid hydrogels. The types of hydrogels are dependent on temperature, concentration of polymer, or $\mathrm{pH}[6,7]$. The high ratio of cPVA caused more adhesive hydrogel in appearance. The results indicated that all hydrogel bases were clear semi-solid. The high ratio of cPVA yielded more mechanical and bioadhesive hydrogel in appearance. The firmness and cohesiveness of the hydrogel decreased when the p407 decreased whereas the cPVA proportion in the formulation increased, where cPVA enhanced bioadhesive of the hydrogel system. In the literature, it was shown that cPVA increased the bioadhesive force of the formulations $[8,9]$.
In addition, the properties of hydrogel can be designed the parameters, including polymer type and molecular weights, polymer concentrations, degree of chemical modification and cross-linked polymer[6,7]. In this study, the properties of hydrogels depend on polymer concentrations. The hydrogel is suitable for herbal extract as water solubility extract, that are steroids, alkaloids, tannins, flavonoids, triterpenes, and glycoside in Thai traditional herb for sport massage gel.

\section{CONCLUSION}

This study found physicochemical properties that the poloxamer-cPVA may be applied to pharmaceutical applications, providing information relating to the ease of removal of a product from a container and the spreadability, besides the adhesive properties. The highlights of this study use complementary techniques to evaluate the physicochemical properties of hydrogel herbal gel in sports massage

\section{ACKNOWLEDGMENTS}

This research was also supported by Thailand Research Fund; PHD/0088/2559 for K. Sukanjana and Thaksin university.

\section{REFERENCES}

[1] S. Kamlungmak, S.Rugmai, K. Tinpun, T . Nakpheng, and $\mathrm{T}$.Srichana, Phase behavior, in vitro drug release, and antibacterial activity of thermoresponsive poloxamer-polyvinyl alcohol hydrogel-loaded mupirocin nanoparticles, Journal of Applied Polymer Science, 2020, pp .49325. DOI :https//:doi.org/10.1002/app.49325

[2] J .L.Soriano-Ruiz, A .C.Calpena-Campmany, M . Silva-Abreu, L.Halbout-Bellowa, N .Bozal-de Febrer, M .J. Rodríguez-Lagunas, and B .ClaresNaveros, Design and evaluation of a multifunctional thermosensitive poloxamerchitosan-hyaluronic acid gel for the treatment of skin burns, International journal of biological macromolecules, 2020, pp .412-422. DOI : https//:doi.org/10.1016/j.ijbiomac.2019.09.113

[3] T .J .Tavakoli and Y .Tang, Mater, Honey/PVA hybrid wound dressings with controlled release of antibiotics :Structural, physico-mechanical and invitro biomedical studies, Materials Science and Engineering, 2017, pp .318-325. DOI : https//:doi.org/10.1016/j.msec.2017.03.272 
]4 [D .Pasqui, M .De Cagna, R .Barbucci, D .Pasqui, M. De Cagna, and R .Barbucci, Polysaccharide-Based Hydrogels :The Key Role of Water in Affecting Mechanical Properties, Polymers, 2012, pp .15171534. DOI :https//:doi.org/10.3390/polym4031517

]5 [A .S .Hoffman, Hydrogels for biomedical applications, Advanced Drug Delivery Reviews, 2012, $\quad$ pp $\quad .18-23 . \quad$ DOI : https//:doi.org/10.3390/gels3010006

]6 [R .Parhi, Cross-linked hydrogel for pharmaceutical applications :a review, Advanced pharmaceutical bulletin, 2017, pp. 515-530. DOI : https//:doi.org/10.15171/apb.2017.064

]7 [Y.Liub, R,Wengb,W.Wangc, X .Weib, J .Lib, X . Chenb, Y .L, F, Lub, and Y, Liab, Tunable physical and mechanical properties of gelatin hydrogel after transglutaminase crosslinking on two gelatin types,
International journal of biological macromolecules,

2020 ,

pp. $\quad 405-413$.

DOI :

https//:doi.org/10.1016/j.ijbiomac.2020.06.185

][[8] N .K .Mongia, K .S .Anseth, and N .A .Peppas, Mucoadhesive poly)vinyl alcohol (hydrogels produced by freezing/thawing processes :applications in the development of wound healing systems, Journal of biomaterials science .Polymer edition, 1996, pp . 1055-64.

DOI : https//:doi.org/10.1163/156856296x00543

S] .9 [Bohrey, V .Chourasiya, and A .Pandey, Polymeric nanoparticles containing diazepam :preparation, optimization, characterization, in-vitro drug release and release kinetic study, Nano convergence, 2016, pp .3. DOI :https//:doi.org/10.1186/s40580-0160061-2 